O. PRZEMYSŁAW MICHOWICZ OFMConv

Wydział Prawa Kanonicznego

Uniwersytetu Papieskiego Jana Pawła II w Krakowie

\title{
ACCEZIONE GIURIDICO-CANONICA DEL SIGNIFICATO DI «TITOLO»
}

Sommario: Premessa. - 1. Evoluzione terminologico-concettuale. - 2. «Titolo» quale facoltà legittimante. - 3. Ambito di nullità matrimoniale. - 4. Ambito di responsabilità. - 5. Ambito di proprietà/fruizione. - Conclusione.

\section{Premessa}

E cosa indubitabile che il termine «titolo» venga usato in diversi accezioni, soprattutto nell'ambito di scienze giuridiche moderne. Il concetto, questo, semanticamente polivalente, si riscontra frequentemente nel linguaggio legale comune sotto diverse diciture quali «titolo di competenza», «titolo di proprietà» o «titolo di responsabilità». Intuitivamente è possibile affermare che il concetto è sinonimo di giusta causa giuridica, necessaria per procedere o per ottenere/difendere un bene, oppure una referenzialità/riconducibilità/ intestazione di qualcosa a qualcuno.

Accanto della vasta gamma di realtà giuridiche avente ad oggetto una concreta titolarità (o meno: nullo titulo), l'ordinamento canonico vigente abbraccia anche varie ipotesi in cui il titulus s'identifica con un nome (di una chiesa, di un'associazione, di un'università), un nome onorifico, o con un ministero sacerdotale in diversi gradi e quant'altro. Ai fini della presente esposizione, non rileva tanto, o solo, la seconda accezione del termine di cui si discorre, ma soprattutto il suo primo significato al quale queste pagine vorrebbero dare una definizione autonoma. Lo studio è volto, dunque, ad una elaborazione concettuale 
del termine nonostante il fatto che la maggior parte degli strumenti generali di ricerca giuridica ignori di trattare ordinariamente questa materia. Il compito che si vuol assolvere consiste quindi in una proposta dottrinale per tracciare un denominatore comune del termine «titolo» partendo dall'analisi teorica di avvenimenti o situazioni previsti dalla fattispecie di una norma canonica o del linguaggio giuridico comune.

\section{Evoluzione terminologico-concettuale}

Entrando nel tema occorre osservare preliminarmente lo sviluppo storico del concetto, specie sotto il profilo semantico. Come sopra osservato, la polisemantica concettuale di titulus ${ }^{1}$ suggerisce che la sua accezione non fosse univoca e come tale sia stata ampiamente e svariatemene impiegata dai giuristi romani. Come documenta uno studio abbastanza arretrato ma carico di forza argomentativa ${ }^{2}$, il significato originario del termine coincideva prevalentemente sia con un inizio/nome/parte di un atto legale, particolarmente quello pretoriale, sia con un'iscrizione di vario genere ${ }^{3}$. Nell'epoca del diritto classico, infatti, il termine equivaleva ugualmente ad un epigrafo, lapido o epitaffio procurato all'occasione di cerimonie funebre o cortei trionfali. Inoltre, il titulus poteva fungere da sinonimo della parola honor e, cioè, doveva essere collegato ad una particolare qualità personale ${ }^{4}$. Dette accezioni del concetto, pur interessantissime e degne di un ulteriore approfondimento, non sono rilevanti molto sotto il profilo dello sviluppo della teoria generale del diritto di cui, in realtà, l'ordinamento canonico è privo.

Quanto all'accezione prettamente giuridica, il medesimo prof. Alvaro d'Ors è di parere che l'impiego del concetto titulus (nel

\footnotetext{
${ }^{1}$ Cfr. I. Palazzini, voce «Titulus», in: Dictionarium morale et canonicum, vol. IV, P. Palazzini (cur.), Romæ 1958, p. 503.

${ }^{2}$ Cfr. A. D’Ors, Titulus, Anuario de Historia del Derecho Español 23 (1953), p. 495-513.

${ }^{3}$ Similmente H.C. BLACK, voce «Title», in: Black's Law Dictionary, $6^{\text {th }}$ ed., St. Paul (Minn.) 1990, p. 1485.

${ }^{4}$ Fornisce numerosi esepmi in dettaglio l'elaborato di D. Aвог Rubio, El concepto jurídico de «título», Cuadernos Doctorales 23 (2009), p. 235-237.
} 
senso per noi ricercato) era estraneo alla comune prassi del diritto romano classico e risale, piuttosto, all'epoca postclassica, e cioè specificatamente all'operato della cancelleria di Diocleziano ${ }^{5}$. Ritenere che i giuristi classici intendessero il termine «titolo» quale motivo o pretesto di base di una situazione giuridica, significa fare un errore, giacché nell'epoca postclassica - contrassegnata da decadenza e da confusione delle fonti ${ }^{6}$ - numerosi testi giuridici subirono delle interpolazioni.

In verità, il passaggio semantico dal titulus-iscrizione al tituluscausa è frutto di un processo lento ma progressivo; si svolse naturalmente in modo tale da non decadere il suo utilizzo nel senso di cartello, insegna, intestazione che fungeva, al contempo, da felice espediente nella redazione dei codici delle leggi o editti pretoriali. La sua prima accezione acquisiva difatti un ulteriore contenuto semantico, quello cioè di personale responsabilità.

A questo proposito, è utile ricordare la descrizione evangelica della crocifissione di Gesù Cristo. Secondo la narrazione di San Marco, il titulus s'identifica con una tavoletta in cui era indicata la causa della condanna insieme al nome del reo $(\mathrm{Mc}, 15,26)^{7}$. Detto impiego derivava, in realtà, dall'accezione primaria ossia era effetto di una popolare prassi di fissare una scritta su un palo mentre si svolgevano le processioni trionfali o funeree. Se ne deduce che il concetto «titolo» con il quale si individuava sia il nome della persona interessata sia i fatti a lui imputabili, col passare del tempo ha acquisito il valore di «causa» o «motivo». Tale connessione rende evidente il legame intercorrente tra causalità e responsabilità (lato sensu) che in questo modo includono nell'unico termine «titolo».

${ }^{5}$ Cfr. D. Авог Rubio, voce «Título», in: J. Otaduy, A. Viana, J. Sedano (curr.), Diccionario General de Derecho Canónico, vol. VII, Navarra 2012, p. 594; A. D’Ors, Titulus..., op.cit., p. 513.

${ }^{6}$ Cfr. A. D’Ors, Titulus..., op.cit., p. 508.

${ }^{7}$ Invece Mt 27, 37: causam ipsiuis scriptam; Lc 23, 38: superscriptio scripta; Gv 19, 19-20: titulum. 
Quanto precede può documentare come i testi giuridici postclassici venissero manipolati a seguito di alterazioni involontarie, mal adattamenti alla realtà $o$ addirittura si riscontravano omissioni di frasi decisive/cruciali presenti nei testi originali. Quest"'attualizzazione" documentaria, non sempre felice, si coglie, tra l'altro, nell'opera chiamata Fragmenta Vaticana in cui si legge: «denuntiari debet et adire prætorem et titulum excusationis expromere» (n. 156). E alquanto logico che l'autore della versione originale intendesse piuttosto l'espressione «causæ excusationis», concordemente al suo impiego del numero precedente ${ }^{8}$.

Per quanto invece riguarda l'opera codiciale di Giustiniano, si deve osservare che il concetto titulus veniva spesso impiegato in vari contesti. Tuttavia, la stragrande maggioranza dei casi d'utilizzo rinviava al significato "causa, pretesto, motivo» ${ }^{9}$ il che autorizza a supporre che il concetto titulus (nel senso di causa) sia un termine di epoca postclassica-bizantina. In realtà, la dottrina osserva che «il iustus titulus usucapionis - da cui derivò il nostro giusto titolo sostituisce la classica iusta causa (...) Non deve poi sfuggire che molte volte i Bizantini possono essersi limitati a sostituire titulus là dove trovarono causa senza fare aggiunte al testo classico, soprattutto senza apportare modifiche sostanziali» ${ }^{10}$.

I dati dell'esposizione che precede autorizzano questa conclusione: l'origine, l'uso e l'esito del termine di cui ci occupiamo, fondamentalmente come sostantivo adoprato al singolare, documentano la funzione di un gruppo di entità (anche giuridiche) oggettive, ma non già la funzione espressiva di una sintesi concettuale riferibile a dette realtà. In effetti, il senso ricercato da questo studio verte strettamente

\footnotetext{
${ }^{8}$ Cfr. P. Krüger, T. Mommsen, W. Studemund (curr.), Collectio librorum anteiustiniani in usum scholarum, vol. 2/3, Berlin 2001, p. 58.

${ }^{9}$ Cfr. R. Von Mayr (cur.), Vocabularum Codicis Iustiniani, vol. I, Hildesheim 1965, coll. 2410-2413.

${ }^{10}$ E. Albertario, Ancora sui glossemi nei Frammenti Vaticani, Studi Giuridici di Diritto Romano 5 (1937), p. 556.
} 
su una pluralità di ragioni giuridiche, cause e motivi legali che potrebbero legittimare un diritto o un'azione da far valere.

\section{2. «Titolo» quale facoltà legittimante}

Il concetto di «titolarità», sia nell'ordinamento giuridico statale sia in quello della Chiesa, pur ampiamente impiegato nei vari contesti, semmai menzionato dai motori di ricerca scientifica, rinvia quasi automaticamente ad una problematica ben diversa, ma tematicamente collegata, ossia all'esercizio del diritto per cui l'accento viene posto quasi sempre sulla netta divisione tra titolarità del diritto e l'esercizio del diritto stesso, come se si trattasse di un momento statico e dinamico del medesimo diritto ${ }^{11}$, e cioè dello ius e dell'actio.

Nella prospettiva che qui interessa il dettato legislativo vigente, seppur indirettamente, prevede delle ipotesi da cui si deduce l'intestazione di un bene (non necessariamente materiale) ad un soggetto a ciò abilitato. Si tratta della disposizione di cui al can. 168 $\mathrm{CIC} / 82$ dove si sancisce la vigenza della proibizione di partecipare alle elezioni gli elettori con doppio titolo personale («etsi quis plures ob titulos ius habet») in ragione della titolarità di due uffici o di una duplice condizione, ognuna delle quali potrebbe abilitarli ad esprimere un voto. La seconda ipotesi si deduce dal can. $951 \mathrm{CIC} / 83$ in cui il Legislatore disciplina, servatis servandis, il principio generale proibente al sacerdote, concelebrante lo stesso giorno una seconda Messa, di percepire - «nullo titulo» - l'offerta.

Quanto all'amministrazione dei beni, va osservato che i canoni 1276,1282 e 1289 CIC/83 adoperano il termine «titolo» in riferimento a qualsiasi causa legittimante un amministratore ad adempiere la propria mansione secondo gli standard espressi nell'astratto modello di un «prudens et diligens pater familias». In specifico, rileva l'ipotesi del can. 1276 CIC/83 che difatti conferma la facoltà di vigilanza e la potestà regolamentare agli ordinari per l'amministrazione di tutti i beni

\footnotetext{
${ }^{11}$ Cfr. voce «Titolarità», in: A. Azarda, E. Eula (curr.), Novissimo Digesto italiano, vol. XIX, Torino 1973, p. 322; V. Frosini, voce «Esercizio del diritto», in: Novissimo Digesto italiano, vol. VI, Torino 1960, p. 823.
} 
ecclesiastici. Lo ius advigilnadi, pur essendo un dovere delegabile, corrisponde ad una responsabilità personale di vigilanza qualora l'amministrazione diretta non proceda correttamente ${ }^{12}$. Il titolare deve pertanto intervenire per ovviare a negligenze o ad abusi ed, eventualmente, per sostituire un tale amministratore ${ }^{13}$. Dette mansioni si evincono dai titoli legittimi attribuiti all'agente modo iure proscripto. Quanto asserito rileva significativamente per una composizione del quadro casistico più complesso, giacché la mancata vigilanza, specie contro le norme legali che la impongono, ad esempio a determinati uffici, potrebbe risultare oggetto d'imputazione di responsabilità non solo a livello civilistico, ma anche penale. La non vigilanza, difatti, da parte di chi esercita un particolare ufficio con doveri istituzionali, tende a promuovere i profili della negligenza grave o, a volte, il mancato impedimento di reati veri e propri (complicità inclusa) ${ }^{14}$.

L'attenta analisi delle norme codiciali permette d'individuare la disposizione legislativa di cui al can. 1407 CIC/83 che disciplina la cosiddetta competenza relativa referenziale alla potestà del giudice ${ }^{15}$. La determinazione di tale competenza in tribunali di prima istanza si regola, in ragione del territorio, sulla base di uno dei diversi titoli che danno luogo a fori cui l'attore può lecitamente aderire, rendendo possibile la citazione in giudizio della parte convenuta secondo quanto prescritto dal diritto. In questo caso, i titoli sono le ragioni/regole che ha il Legislatore di attribuire, in via ordinaria, la cognizione delle cause ad un certo tribunale e non ad un altro ${ }^{16}$. I titoli rispondono, dunque, ad una scelta legislativa che investe questioni sostanziali in

\footnotetext{
${ }^{12}$ Cfr. C. Begus, Diritto patrimoniale canonico, Città del Vaticano 2007, p. 167-168.

${ }^{13}$ Cfr. M. López Alarcón, Sub. can. 1276 CIC, in: Codice di Diritto Canonico e leggi complementari, J.I. Arrieta (cur.), Roma 2007, p. 844.

${ }^{14}$ Cfr. P. Gherri, Titoli di responsabilità dei Superiori generali degli IVC in ambito extracanonico, Commentarium pro Religiosis 95 (2014), p. 50.

${ }^{15} \mathrm{Cfr}$. anche can. $1411 \mathrm{CIC} / 83$ che contempla la problematica del titolo del contratto che dà origine a tre diversi fori concorrenziali.

${ }^{16}$ Cfr. C. De Diego Lora, Sub can. 1407 CIC/83, in: Codice di Diritto Canonico e leggi complementari, J.I. Arrieta (cur.), Roma 2007, p. 844; M.J. Arroba Conde, Diritto processuale canonico, $5^{\text {a }}$ ed., Roma 2006, p. 106.
} 
modo che diventino criteri specifici ed immediati che abilitano un giudice ad accettare e portare a termine (o meno) una controversia contenziosa o penale che sia ${ }^{17}$.

Infine, è opportuno menzionare il contenuto normativo di cui al can. 1497, \$2 CIC/83 che disciplina l'esercizio di azioni cautelari all'interno del processo canonico. Secondo il Codice vigente sono tre le ipotesi che permettono l'attuazione di tali misure; qui rileva solo quella inerente l'effettiva dimostrazione della titolarità di un credito in attesa di adempimento. Ė lampante che il titulus si riferisca ad una posizione ordinamentale attribuente ad un soggetto lo ius ad rem e, conseguentemente, la rispettiva actio ( $v$. supra).

Se non ci inganniamo, siamo giunti alla soglia del problema che ci interessa di impostare e risolvere: la determinazione del concetto titulus in senso strettamente giuridico-canonico. Dall'esposizione che precede risulta che in tutte le summenzionate ipotesi sorgono situazioni soggettive vere e proprie abilitanti un soggetto legittimato dall'ordinamento canonico ad usufruire quanto precluso nell'oggetto della propria prerogativa personale (bene giuridico), non dovendo però corrisponderne.

Ora, ai fini della complementarietà metodologica del presente elaborato, è necessario addentrarsi nel tema del linguaggio giuridico dal quale - vista la limitatezza di queste pagine - vengono analizzati solo alcuni esempi: «titolo di nullità matrimoniale», "titolo di responsabilità» e «titolo di proprietà/fruizione».

\section{Ambito di nullità matrimoniale}

Il consensus che facit nuptias è da considerarsi nel senso ordinario della parola di consenso, accordo momentaneo di due volontà producente un vincolo obbligatorio perenne che si conserva tale sebbene quelle volontà divengano poi discordi - come nei contratti in genere servatis servandis - i quali hanno appunto per precipuo scopo quello di assicurare lo scambio dei servigi umani contro la

\footnotetext{
${ }^{17}$ Cfr. J. OchoA, I titoli di competenza, in: AA. Vv., Il processo matrimoniale canonico, collana Studi Giuridici t. XVII, Città del Vaticano 1994, p. 133.
} 
mutabilità del volere umano. In teoria, quello richiesto per le nuptice è un consenso consistente in un accordo di volontà istantaneo, non continuato, senza alcun limite temporale quanto alla sua durata (tranne la morte) ${ }^{18}$. E, dunque, consenso il momento generante di una nuova condizione canonica degli sposi, quella cioè che implica sia la loro idoneità ad essere titolari di diritti e doveri specifici sia la capacità di porre validamente in essere atti idonei ad incidere sulle situazioni giuridiche (di cui si è titolari). Detto altrimenti: si tratta di un'abilità giuridica che corrisponde ad una capacità di agire che un coniuge, rispondente alle condizioni prefissate dal can. $96 \mathrm{CIC} / 83$, acquisisce nel sacramento del matrimonio ${ }^{19}$. In verità, il can. 1674 CIC/83 stabilisce l'abilità requisita ad avviare l'esame di una causa di nullità matrimoniale o meglio, determina la capacità di agire specifica di chi può impugnare un matrimonio ${ }^{20}$. È doveroso osservare che la normativa vigente non impedisce ai coniugi acattolici o scomunicati d'accusare il proprio matrimonio, ciò in conformità alla prassi ormai consolidata dopo la pronuncia autentica della Commissione Pontificia per l'interpretazione del Codice di diritto canonico del $1973^{21}$.

Paradossalmente, chi vuole dichiarare il proprio matrimonio nullo deve mettere in discussione la validità dello stesso sacramento qui intesa, questa legittimità, come elemento generante il primo presupposto giuridico necessario per esserne titolare, cioè lo status canonico del coniuge. È opportuno ricordare che l'invalidità del matrimonio canonico non si presume né dipende da una certezza soggettiva o mera opinione di un coniuge circa le nozze celebrate

\footnotetext{
${ }^{18}$ Cfr. J.F. Castaño, Il sacramento del matrimonio, $2^{\text {a }}$ ed., Roma 1992, p. 41-43.

${ }^{19}$ Cfr. P. VAldrini, Comunità, Persone, Governo. Lezioni sui Libri I e II del CIC 1983, Città del Vaticano, 2013, p. 155.

${ }^{20}$ Cfr. F. Daneels, Il diritto di impugnare il matrimonio, AA. Vv., Il processo matrimoniale canonico, collana Studi Giuridici t. XVII, Città del Vaticano 1994, p. 393-394.

${ }^{21}$ Cfr. Pontificia Commissio Codici Iuris Canonici Authenitice InterpreTANDO, «Utrum ... vigeat adhuc responsum S.S.C.S. Officii diei 27 iannuarii 1928», AAS 62 (1973), p. 59. In questo caso è ancora discutibile la questione della deroga alla disposizione del can. $96 \mathrm{CIC} / 83$.
} 
(can. 1100 CIC/83), non essendo, un matrimonio, contratto meramente privatistico. Il processo di nullità matrimoniale è caratterizzato, infatti, da una forte impronta pubblicista, senza diminuire però il protagonismo specifico dei coniugi in quanto titolari del rapporto coniugale in discussione. In tal senso la presunzione generale sulla validità matrimoniale è attenuata dalla possibilità di provare il contrario. Segue che il processo di nullità risponde al diritto del singolo fedele di contrarre un valido matrimonio (can. $1058 \mathrm{CIC} / 83)^{22}$.

Or dunque, data per certa la vigenza del principio concernente la presunzione di validità di qualsiasi atto giuridico (cann. 124, $\$ 2$ e $1060 \mathrm{CIC} / 83$ ), specie quello matrimoniale, ai fini della piena e legittima esecuzione della dichiarazione di nullità, è necessaria inoltre la sussistenza delle circostanze (gli impedimenta, i vizi del consenso, la mancanza della forma canonica) in veste di fatti e prove quali condizioni di cose e di tempo, giuridicamente rilevanti. Se l'oggetto del giudizio contenzioso sono i fatti giuridici da dichiarare o situazioni giuridiche meritevoli di dichiarazioni o riconoscimenti giurisdizionali (can. $1400 \mathrm{CIC/83),} \mathrm{chi} \mathrm{invoca} \mathrm{tale} \mathrm{garanzia} \mathrm{giudiziale}$ deve dimostrare e far palese la fondatezza del fumus boni iuris adempiendo, al contempo, delle esigenze poste dal diritto, cioè i presupposti processuali. Infine, deve rivestire i requisiti formali stabiliti dal Legislatore ${ }^{23}$.

Nelle cause di nullità matrimoniale la legittimazione processuale, intesa come capacità d'instaurare legittimamente l'azione deriva, dunque, dalla volontà contrattuale/negoziale la quale indica la fonte di tale prerogativa di contestazione, esercitabile poi a modo di peculiari mezzi dello stesso ius appellandi previsti dal diritto positivo quali querela di nullità, appello o ricorso (restitutio in integrum). Quest'azione si concretizza, di fatto, nel diritto di impugnare il matrimonio.

\footnotetext{
${ }^{22}$ Cfr. M.J. Arroba Conde, Diritto processuale canonico, op. cit., p. 599.

${ }^{23}$ Cfr. C. De Diego Lora, Sub can. 1400 CIC/83, in: Codice di Diritto Canonico e leggi complementari, J.I. Arrieta (cur.), Roma 2007, p. 932.
} 


\section{Ambito di responsabilità}

La problematica della titolarità in ambito di questioni connesse alla vasta tematica di responsabilità è molto più complessa rispetto all'appena analizzato tema della nullità matrimoniale in genere; questo, fra l'altro, per un semplice motivo secondo il quale l'espressione «titolo di responsabilità» cambia significato secondo la qualità giuridica di atti o fatti. In altre parole: in campo penale detta locuzione si riferisce alla graduazione della partecipazione ai reati in concorso, mentre in ambito civile corrisponde ad una conseguenza civilistica, di solito economica, alternativa o accessoria a quella penale.

Prima facie, la responsabilità penale è conseguenza di un rapporto causa-effetto sottostante alla dinamica del danno-risarcimento ovverosia secondo la teoria tripartita secondo cui il fatto delitto si consuma allorché risultino integrati tre distinti presupposti: la tipicità, la valutazione dell’antigiuridicità e la colpevolezza. Nella giusta calibrazione del fatto delittuoso, indipendentemente da quale sia la tesi seguita nella sua strutturazione, centrale è la riflessione sull'idea di colpevolezza. Nel linguaggio del diritto penale (perlomeno italiano), la colpevolezza abbraccia tutti i presupposti ed i requisiti necessari alla correlativa affermazione, sia questi oggettivi sia quelli soggettivi. In un'accezione più ristretta, invece, la colpa in senso lato significa l'insieme degli elementi solo soggettivi sui quali si fonda la responsabilità, imputabilità compresa ${ }^{24}$.

La nozione di responsabilità penale indica la relazione tra un fatto ed un soggetto che è rivestita, detta relazione, della specifica disapprovazione cui la legge connette l'applicabilità in astratto di una sanzione penale, laddove la sua applicazione in concreto sia concernente la teoria della condanna ${ }^{25}$. Si tratta dell'ascrivibilità

\footnotetext{
${ }^{24}$ Cfr. G. VAssalli, voce «Colpevolezza», in: Enciclopedia giuridica, vol. VI, Torino 1988, p.1.

${ }^{25}$ Cfr. A. Fiorella, voce « Responsabilità penale», in: Enciclopedia giuridica, vol. XXIX, Torino 1988, p.1289.
} 
dell'evento con il previo accertamento del nesso causale tra il fatto stesso e l'agente sanzionato ${ }^{26}$.

Quanto poi al diritto penale canonico, il concetto di responsabilità giuridica - presupposto per l'applicabilità delle pene previste - si distingue concettualmente dall'imputabilità giuridica ${ }^{27}$. Mentre il secondo termine si riferisce all'atto delittuoso attribuibile ad un soggetto che l'ha compiuto con coscienza e volontà ${ }^{28}$, la responsabilità invece, specifica che l'agente debba rispondere delle conseguenze. L'imputabilità, pur indicando la proprietas obiectiva actionis, nella prassi è usata come termine per designare una qualità dell'agente, cioè funge da elemento subiettivo del delitto nelle forme del dolo e della colpa, comprensiva anche della colpevolezza attinente alla sfera morale di chi ha compiuto una violazione esterna di una legge o di un precetto.

Il can. 1321, $\$ 3$ CIC/83 stabilisce la presunzione dell’imputabilità nel senso che essa stessa notoriamente comprende sia il titolo di dolo che della colpa. In altri termini: la presunzione d'imputabilità equivale alla presunzione della libertà dell'atto che ha violato una norma, sia che tale violazione sia ex dolo o ex culpa $a^{29}$. Tutto questo significa che la consumazione di un delitto ed eventuale punizione, non può avvenire senza la materializzazione di un atto umano (causa-effetto).

Ricercando l'accezione del «titolo di responsabilità penale», accanto al modello tradizionale di responsabilità a titolo di colpa, esiste anche un'ipotesi concorrente cui presupposti però non comprendono né le condotte, né i fatti, e neanche le conseguenze negative su terzi

\footnotetext{
${ }^{26}$ Questo studio si limita all'ipotesi della responsabilità soggettiva, poiché il Legisltore canonico non prevede nella sua opera le ipotesi della responsabilità oggettiva e quella per fatto altrui. Cfr. H. PreE, voce «Responsabilidad de la administración», in: J. Otaduy, A. Viana, J. Sedano (curr.), Diccionario General de Derecho Canónico, vol. VI, Navarra 2012, p. 987.

${ }^{27}$ Cfr. A. D'Auria, L'imputabilità nel diritto penale canonico, Roma 1997, p. 54.

${ }^{28}$ Cfr. C.V. Mancini, L'elemento intenzionale nella teoria canonistica del reato, Torino 2001, p. 18.

${ }^{29}$ Cfr. V. De Paolis, D. Cito, Le sanzioni nella Chiesa. Commento al Codice di dirtto canonico. Libro VI, Roma 2008, p. 153.
} 
o un'eventuale modalità del risarcimento. L'elemento ricercato, invece, siano le cause o le modalità di coinvolgimento passivo di un soggetto in atti o fatti altrui. Quanto al «titolo di responsabilità» il requisito rilevante dovrebbe essere una mera ed effettiva possibilità di rispondere senza compiere nessun tipo di atto penalmente (o meno) rilevante. In verità, si tratta della determinazione di una situazione con un'irriducibile prevalenza degli elementi oggettivi e fattuali rispetto a qualunque apporto soggettivo ${ }^{30}$. Detto altrimenti: il titolo di responsabilità, secondo tale significato, coincide col coinvolgimento soggettivo puramente formale e, cioè, sorge solo per mere ragioni giuridiche, non invece per qualsiasi partecipazione attiva. In tal senso, considerando l'ipotesi del diritto penale canonico, diventa possibile la determinazione degli eventuali motivi della graduazione nella partecipazione ai reati in concorso (ad. es. ipotesi della complicità accessoria) $^{31}$.

\section{Ambito di proprietà/fruizione}

Il diritto di proprietà, pur essendo quella facoltà che forse più di qualsiasi altro diritto interpreta uno dei fondamentali bisogni dell'uomo ${ }^{32}$, non è tuttavia una forma di sovranità assoluta sui beni. È piuttosto un diritto che s'inserisce armonicamente nel più ampio ambiente sociale in modo che non contrasta col pieno godimento e con la libera disposizione degli stessi beni.

Ricollegandosi alle premesse del presente lavoro, l'argomento che interessa di più sono i presupposti giuridici della valida trasmissione di proprietà e, conseguentemente, anche fruizione, salva fatta l'osservazione che lo ius fruendi non sempre implica la sussistenza di qualunque forma di proprietà legalmente acquisita (enfiteusi).

\footnotetext{
${ }^{30}$ Cfr. P. Gherri, Titoli di responsabilità dei Superiori generali, op. cit., p. 34.

${ }^{31}$ Cfr. A. Maroza, Sub. can. 1329, in: A. Marzoa, J. Miras, R. Rodríguez-Ocaña (curr.), Comentario exegético al Código de derecho canónico, vol. IV/1, Pamplona, 1997, p. 344.

${ }^{32}$ Cfr. Tribunal Apostolicum Romanæ Rotæ, coram Palestro, decisio diei 31 ianuarii 1990, in: Rotæ Romanæ Decisiones, vol. LXXXII, p. 61, nº 9.
} 
Essendo la proprietà un bene del tutto specifico, particolarmente quanto alle facoltà tramite le quali si esprime detto diritto, la sua trasmissione da un soggetto ad un altro non deve sempre presupporre un atto di volontà emesso da un soggetto giuridicamente abile e a ciò capace $^{33}$ (ad es. la trasmissione testamentaria o ereditaria). La maggior parte degli ordinamenti civili prevedono, infatti, varie modalità d'acquisto quali documento statale, contratto (di compravendita, donazione), sentenze dei tribunali, decisioni degli organi amministrativi competenti, etc. Si tratta, dunque, di una fonte della facultas proprietatis che crea, di conseguenza, un nesso di natura giuridica tra un bene ed un soggetto a ciò giuridicamente preposto, sebbene lo stesso legame potrebbe essere meramente formale.

Lungi dall'addentrarsi nella problematica inerente una soluzione ormai tendenzialmente univoca al problema del soggetto titolare del diritto di proprietà ${ }^{34}$, qui è sufficiente aderire all'affermazione, dottrinalmente condivisibile, secondo la quale la proprietà nel diritto canonico sia attribuita secondo i criteri di diritto privato. Tale modalità d'acquisto non vieta che si possa qualificare una proprietà come pubblica, stante la norma del can $1256 \mathrm{CIC} / 83$, poiché si tratta dei beni di persone giuridiche pubbliche ${ }^{35}$. Per acquisto si deve intendere non solo l'atto o il fatto giuridico avente la conseguenza di rendere l'acquirente titolare di un diritto di proprietà sui beni, ma anche l'atto o il fatto giuridico i cui effetti portano alla titolarità di altri diritti minori (ad es. quello di obbligazione) con le relative facoltà ${ }^{36}$. Si badi bene che il Legislatore canonico ha scelto di definire i beni ecclesiastici non sulla base dei fini che giustificano la loro proprietà da parte della

\footnotetext{
${ }^{33}$ Cfr. Tribunal Apostolicum Romanæ Rotæ, coram Pompedda, decisio diei 8 novembris 1983, in: Rotæ Romanæ Decisiones, vol. LXXV, p. 577, nº 4.

${ }^{34}$ Cfr. C. Begus, Diritto patrimoniale canonico, op. cit., p. 35-37.

${ }^{35}$ Cfr. J.T. Martín de Agar, Bienes temporales y missíon de la Iglesia, in: Aa.Vv., Manual de Derecho Canónico, Pamplona 1991, p. 705-706.

${ }^{36}$ Cfr. F. Coccopalmerio, Diritto patrimoniale della Chiesa, in: AA.Vv., Il diritto nel mistero della Chiesa, vol. IV (collana Quaderni di Apollinaris 4), Roma 1980, p. 26.
} 
Chiesa, ma usando come criterio divaricante il soggetto titolare dei beni stessi, vista la scelta già compiuta nel 1917 (can. 1497, \$2 CIC/17).

L'ipotesi dell'acquisto del titolo è data anche dall'istituto della prescrizione (can. 197 CIC/83). La giurisprudenza dei tribunali ecclesiastici riconosce la prescrizione come modo recepito dalla Chiesa e stabilito dal diritto positivo per l'acquisto di un diritto reale o per la perdita di un diritto a causa del trascorre del tempo e ne distingue, similmente così come il Codice vigente, due fattispecie: quella acquisitiva (usucapione) e quella liberativa ${ }^{37}$. È importante rilevare che la giurisprudenza definisce il titolo quale «causa, natura sua habilis ad trasferendum dominium $»^{38}$. Si tratta, dunque, di una ragione astrattamente idonea a trasferire la proprietà di una cosa $\mathrm{e}$ fondante il giudizio del possessore il quale deve ritenere che la cosa posseduta sia giustamente sua. In altre parole: è una «causa que sirve de base o que justifica una relación o situación jurídica» ${ }^{39}$, di solito di vantaggio goduta da un soggetto e tutelata dal diritto positivo.

\section{Conclusione}

L'argomento dell'accezione di significato di «titolo» nell'ordinamento canonico è stato affrontato nella consapevolezza che «tutto che al mondo è civile ... è romano ancora» - come osservava Giosuè Carducci nelle Odi Barbare - : pertanto la concezione del titulus, nel suo sviluppo e nei suoi elementi, è stata analizzata in modo approfondito soprattutto attraverso la rilettura critica del testo legislativo vigente e del linguaggio giuridico corrente.

Questo itinerario di ricerca ha esplicitato la distribuzione del lavoro su cinque punti ognuno del quale aveva ad oggetto diverse ipotesi dell'impiego terminologico della parola «titolo». Lungo detto percorso

\footnotetext{
${ }^{37}$ Cfr. Tribunal Apostolicum Sacræ Romanæ Rotæ, coram Palazzini, decisio diei 24 octobris 1973, in: Rotæ Romanæ Decisiones, vol. LXV, p. 674, nº 6.

${ }^{38}$ Cfr. Tribunal Apostolicum Sacræ Romanæ Rotæ, coram Solieri, decisio diei 22 iunii 1923, in: Rotæ Romanæ Decisiones, vol. XV, p. 123, n 13.

${ }^{39}$ F. Gutiérrez-Alviz y Armario, voce «Titulus», in Diccionario de derecho romano, Madrid 1982, p. 669.
} 
si è rilevato l'estrema importanza dei presupposti giuridici quali veri contrassegni della corretta determinazione di un possibile modello astratto del termine. La sua costruzione teorica permette di constatare che per «titolo giuridico» si deve intendere ogni ragione giuridica per cui un soggetto gode di una facoltà autorizzante al compimento di un atto oppure trattasi di una intestazione o referenzialità di un atto o un fatto ad una persona; una riconducibilità, anche meramente formale, a prescindere cioè dall'elemento soggettivo di volontà.

L'indagine fin qui svolta consente di affermare, inoltre, che detto concetto è un felice espediente determinante una vasta gamma di cause, motivi, pretesti, principi, fondamenti di base che - avvalorati di determinati condizioni di tempo e di spazio - dimostrano l'origine e/o la fonte di una prerogativa personale o individuano le modalità concrete d'acquisto di un diritto insieme alle loro caratteristiche proprie.

\section{Canonical meaning of legal term «title»}

The main aim of the present paper was to determine correctly legal significance of the term «title». The research started by analyzing the roman law, specifically those fragments which confirmed the doctrine's intuition concerning the fact that the strictly legal connotation of the term should be dated under the regime of Diocletian.

It was also highlighted that the Code of Canon Law 1983 uses the notion in different meanings from which the most researched was this, which embraces all legal categories, included those existing in spoken legal language. Eventually, three dimensions of commercium iuridicum: matrimonial, the ones related to an idea of liability and propriety, were indicated as abstract models containing a possible definition of the concept.

SŁOWA KLUCzowe: «tytuł»; tytulariusz; prawo do zaskarżenia małżeństwa; tytuł odpowiedzialności; tytuł właśności

KEY WORDS: «title»; titularity; right to declare matrimony null; liability title; propriety title 
Nota o Autorze:

O. DR PrzemysŁaw Michowicz OFMConv, franciszkanin należący do krakowskiej Prowincji Zakonu Braci Mniejszych Konwentualnych, asystent na Wydziale Prawa Kanonicznego UPJPII, wykładowca prawa kanonicznego i prawa wyznaniowego w Wyższym Seminarium Duchownym Franciszkanów w Krakowie. 